\title{
Effective Exercises for Targeting the Gluteus Medius
}

\begin{abstract}
Karrie L. Hamstra-Wright and Kellie Huxel Bliven
Clinical Scenario: The gluteus medius (GM) is thought to play an important role in stabilizing the pelvis and controlling femoral adduction and internal rotation during functional activity. GM weakness, resulting in decreased stabilization and control, has been suggested to be related to lower extremity dysfunction and injury. Many clinicians focus on strengthening the GM to improve lower extremity kinematics for the prevention and rehabilitation of injury. An indirect way to measure GM strength is through electromyography. It is generally assumed that exercises producing higher levels of activation will result in greater strengthening effects. ${ }^{3}$ Understanding what exercises result in the greatest level of GM activation will assist clinicians in their injury prevention and rehabilitation efforts. Focused Clinical Question: In a healthy adult population, what lower extremity exercises produce the greatest mean GM activation, expressed as a percentage of maximum voluntary isometric contraction?
\end{abstract}

Keywords: electromyography, exercise, hip, strength

\section{Clinical Scenario}

The gluteus medius (GM) is thought to play an important role in stabilizing the pelvis and controlling femoral adduction and internal rotation during functional activity. ${ }^{1,2} \mathrm{GM}$ weakness, resulting in decreased stabilization and control, has been suggested to be related to lower extremity dysfunction and injury. ${ }^{1,2}$ Many clinicians focus on strengthening the GM to improve lower extremity kinematics for the prevention and rehabilitation of injury. An indirect way to measure GM strength is through electromyography (EMG). It is generally assumed that exercises producing higher levels of activation will result in greater strengthening effects. ${ }^{3}$ Understanding what exercises result in the greatest level of GM activation will assist clinicians in their injury prevention and rehabilitation efforts.

\section{Focused Clinical Question}

In a healthy adult population, what lower extremity exercises produce the greatest mean GM activation, expressed as a percentage of maximum voluntary isometric contraction (MVIC)?

Hamstra-Wright is with the Dept of Kinesiology and Nutrition, University of Illinois at Chicago, Chicago, IL. Huxel Bliven is with the Dept of Interdisciplinary Health Sciences, A.T. Still University, Mesa, AZ.

\section{Summary of Search, "Best Evidence" Appraised, and Key Findings}

- The literature was searched for studies with a level of evidence 4 or higher that examined which lower extremity exercises produced the greatest mean GM activation expressed as a percentage of MVIC in healthy adults.

- The literature search produced 5 cross-sectional studies for inclusion; these were the only studies found that met all inclusion and exclusion criteria.

- Each study examined a variety of exercises including weight-bearing (WB), non-weight-bearing (NWB), single-leg, and double-leg exercises.

- Collectively, the exercises producing the greatest GM activation (in no specific order) were the single-leg squat, single-leg wall squat, pelvic drop, side bridge, and side-lying hip abduction.

\section{Clinical Bottom Line}

In a healthy population, there is minimal evidence to support the use of the single-leg squat (with or without wall support), pelvic drop, side bridge, and side-lying hip-abduction exercises when aiming to maximize GM activation. These exercises produced the greatest mean GM activation in the 5 studies reviewed in this article. However, the quality of the evidence is low given the cross-sectional designs of these studies. It is difficult to strongly suggest these exercises given the quality of evidence, but based on the current literature available these are the exercises that appear to result in the greatest GM activation. If used, consideration should be given to issues 
such as injury history, functional ability, and strength of the surrounding core muscles when selecting exercises to implement and progress.

Strength of Recommendation: There is grade C evidence ${ }^{4,5}$ that the WB exercises single-leg squat, singleleg wall squat, pelvic drop, and side bridge, in addition to NWB side-lying hip abduction, result in the greatest GM activation.

\section{Search Strategy}

\section{Terms Used to Guide Search Strategy}

- Patient/Client group: adult or active or healthy

- Intervention/Assessment: gluteus medius and exercise

- Comparison: not applicable

- Outcome: acti* or activation or muscle activation or EMG or electromyography

\section{Sources of Evidence Searched}

- The Cochrane Library

- PEDro Database

- Medline

- CINAHL

- SPORTDiscus

- Additional resources obtained via hand search

\section{Inclusion and Exclusion Criteria}

\section{Inclusion Criteria}

- Studies that compared mean GM activation between 2 or more WB or NWB exercises

- Limited to the English language

- Limited to studies that reported mean EMG signal amplitudes of the GM normalized to MVIC

- Limited to the last 11 years (2000-2010)

\section{Exclusion Criteria}

- Studies that included individuals younger than 18 years and older than 65 years

- Studies that included currently injured individuals

- Studies that measured GM subdivisions versus the GM as a whole

- Studies that compared aquatic exercises

- Studies that included an intervention other than, or in addition to, exercise (eg, vibration, orthotics, tape)

\section{Results of Search}

Five relevant studies were located and categorized as shown in Table 1 (based on Levels of Evidence, Centre for Evidence Based Medicine, 2009).
Table 1 Summary of Study Designs of Articles Retrieved

\begin{tabular}{llll}
\hline $\begin{array}{l}\text { Level of } \\
\text { evidence }\end{array}$ & Study design & $\begin{array}{l}\text { Number } \\
\text { located }\end{array}$ & Reference \\
\hline 4 & $\begin{array}{l}\text { Cross- } \\
\text { sectional }\end{array}$ & 5 & $\begin{array}{l}\text { Ayotte et } \mathrm{al}^{6} \\
\text { Bolgla and } \mathrm{Uhl}^{7} \\
\end{array}$ \\
& & Boudreau et $\mathrm{al}^{8}$ \\
& & Distefano et $\mathrm{al}^{9}$ \\
& & Ekstrom et al ${ }^{10}$ \\
\hline
\end{tabular}

\section{Best Evidence}

The studies in Table 2 were identified as the best evidence and selected for inclusion in this critically appraised topic (CAT). Reasons for selecting these studies were that they compared GM activation in a healthy population between 2 or more exercises that were WB or NWB and the main outcome reported was mean GM activation expressed as a percentage of MVIC.

\section{Implications for Practice, Education, and Future Research}

GM activation occurred during all the exercises reviewed in this article, but to different degrees. Activation was greatest in the following exercises, ranked in descending order: side-lying hip abduction $(81 \% \pm 42 \%$ MVIC $),{ }^{9}$ side bridge $(74 \% \pm 30 \% \mathrm{MVIC}),{ }^{10}$ single-leg wall squat $(72 \% \pm 22 \% \mathrm{MVIC}){ }^{6}$ single-leg squat $(64 \% \pm 24 \%$ MVIC and $30 \% \pm 9 \%$ MVIC), ${ }^{8,9}$ pelvic drop $(57 \% \pm$ $32 \%$ MVIC), ${ }^{7}$ unilateral bridge $(47 \% \pm 24 \%$ MVIC $),{ }^{9}$ WB with flexed hip abduction $(46 \% \pm 34 \%$ MVIC $),{ }^{7}$ forward step-up $(44 \% \pm 17 \% \mathrm{MVIC}),{ }^{6}$ and lunge (19\% $\pm 12 \%$ MVIC) ${ }^{8}$

For muscle-strength adaptations to occur, Andersen et $\mathrm{al}^{3}$ suggest that neuromuscular activation be in the range of $40 \%$ to $60 \%$ of maximal effort. Most of the exercises identified herein fall within this range, indicating that they are sufficient exercises for strengthening the GM. The 2 exercises outside this range are the lunge, which activated the nondominant GM at $19 \%$ of its MVIC, and the single-leg squat reported by Boudreau et $\mathrm{al}^{8}{ }^{8}$ which activated the GM at $30 \%$ of its MVIC. However, the single-leg squat reported by Distefano et $\mathrm{al}^{9}$ activated the GM at $64 \%$ of its MVIC. Reasons for this large discrepancy may be differences in how the squat was performed or how MVIC was measured. In the Distefano et $\mathrm{al}^{9}$ study, participants performed the single-leg squat by flexing until they could touch their contralateral middle finger to the outside of their WB foot without reaching with the shoulder. This may have posed a greater challenge in frontal-plane stability and thus increased GM activation in contrast to the participants in the Boudreau et $\mathrm{al}^{8}$ study, who were instructed to squat down as far as possible and return to the starting position without losing 


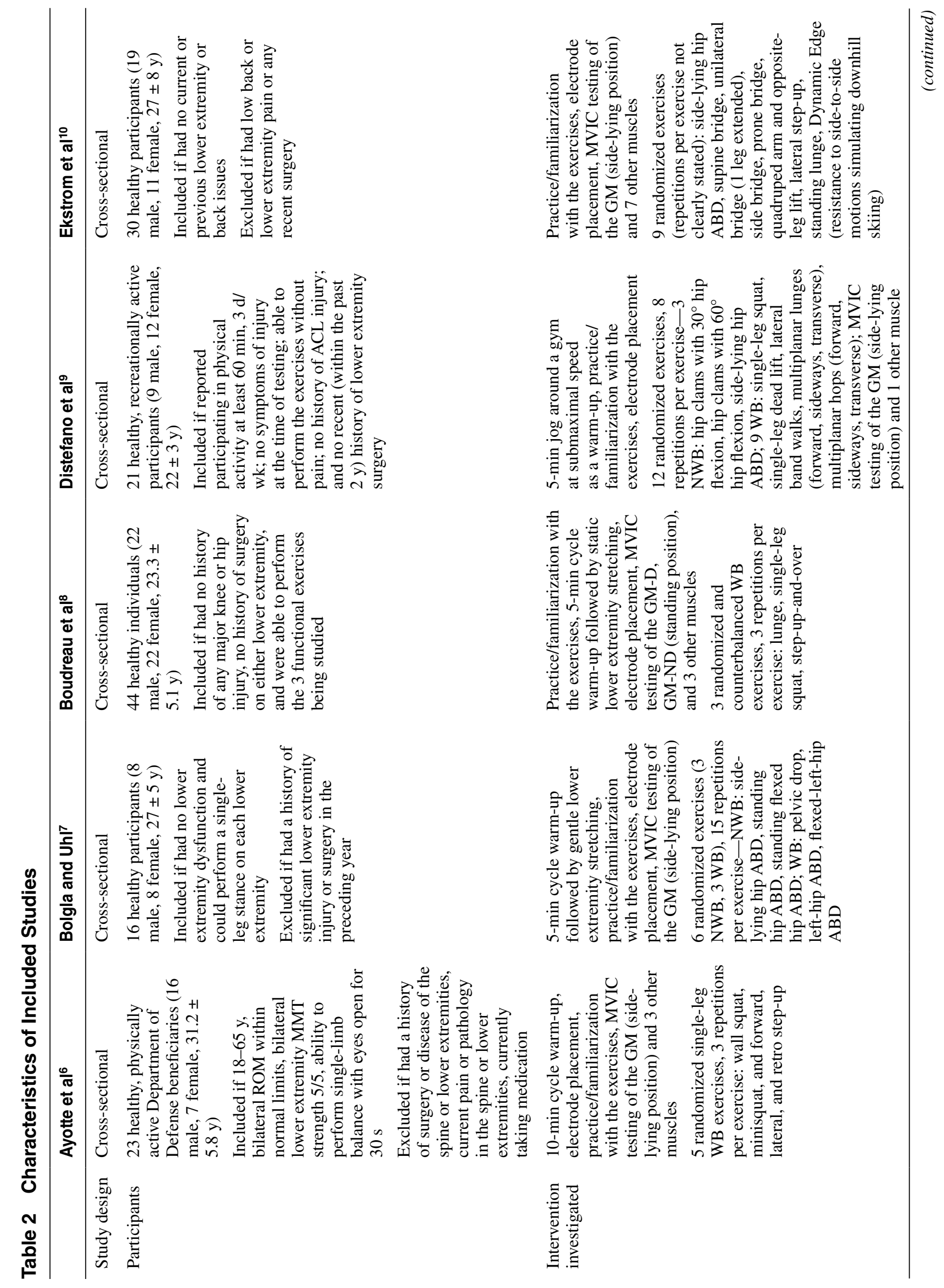







their balance. Furthermore, Boudreau et $\mathrm{al}^{8}$ performed GM MVIC testing in a standing position, which may not have isolated the GM as well as the more commonly used side-lying position used by Distefano et al. ${ }^{9}$

Clinicians implementing exercises to strengthen the GM should consider incorporating the exercises identified in this CAT that fall within the range of $40 \%$ to $60 \%$ MVIC (side-lying hip abduction, side bridge, single-leg wall squat, single-leg squat, pelvic drop, unilateral bridge, WB with flexed-hip abduction, and forward step-up). Exercise selection should be based on various factors such as the patient's functional abilities, injury status, and overall core strength. For example, in patients rehabilitating a hip-abductor injury, it may be appropriate to begin with an exercise that requires less GM activation, such as the lunge, before progressing to exercises requiring greater activation. Similarly, depending on the patient's WB ability and biomechanics, clinicians may want to consider using the NWB exercises, particularly side-lying hip abduction, before progressing to the WB exercises.

It is important to note that EMG may not be a clinically relevant tool because of its cost, time involved, expertise required, and methodological limitations. If EMG is available for clinical use, careful placement of the surface electrodes is important to reduce variability within and across patients, which may decrease the likelihood of cross-talk between muscles. Cross-talk can particularly be an issue due to the proximity of the gluteus maximus and medius. ${ }^{10}$ In addition, although the relationship between EMG amplitude and muscle force is thought to be generally linear during isometric contractions, ${ }^{11}$ EMG is not a direct measure of strength and should not be used in isolation to monitor strength gains. Rather, EMG provides clinicians with insight regarding the level of activation of a particular muscle so a judgment can be made regarding the utility of the exercise to target the desired muscle during strengthening exercises. ${ }^{10}$

This CAT informs clinical practice by identifying which current and commonly used strengthening exercises are thought to most activate the GM. However, the lack of high-quality research investigating GM activation during various rehabilitative exercises highlights the need for prospective and randomized controlled trials that examine GM activation and strength gains over time in healthy and injured populations. Furthermore, future investigations examining the role of the GM subdivisions during preventive and rehabilitative exercises targeting the GM may also be clinically relevant. Limited research indicates that the anterior, middle, and posterior subdivisions of the GM may have different, yet synergistic, actions. ${ }^{12}$ This CAT should be reviewed in 2 years to determine whether there is additional best evidence that may change the clinical bottom line for this clinical question.

\section{References}

1. Cichanowski HR, Schmitt JS, Johnson, RJ, Niemuth PE. Hip strength in collegiate female athletes with patellofemoral pain. Med Sci Sports Exerc. 2007;39:1227-1232.

2. Fredricson M, Cookingham CL, Chaudhari AM, Dowdell BC, Oestreicher N, Sahrmann SA. Hip abductor weakness in distance runners with iliotibial band syndrome. Clin J Sport Med. 2000;10:169-175.

3. Andersen LL, Magnusson SP, Nielsen M, Haleem J, Poulsen K, Aagaard P. Neuromuscular activation in conventional therapeutic exercises and heavy resistance exercises: implications for rehabilitation. Phys Ther. 2006;86:683-697.

4. McKeon PO, Medina JM, Hertel J. Hierarchy of research design in evidence-based sports medicine. Athl Ther Today. 2006;11:42-45.

5. Medina JM, McKeon PO, Hertel J. Rating the levels of evidence in sports-medicine research. Athl Ther Today. 2006; 11:45-48.

6. Ayotte NW, Stetts DM, Keenan G, Greenway EH. Electromyographical analysis of selected lower extremity muscles during 5 unilateral weight-bearing exercises. $J$ Orthop Sports Phys Ther. 2007;37:48-55.

7. Bolgla LA, Uhl TL. Electromyographic analysis of hip rehabilitation exercises in a group of healthy subjects. $J$ Orthop Sports Phys Ther. 2005;35:487-494.

8. Boudreau SN, Dwyer MK, Mattacola CG, Lattermann C, Uhl TL, McKeon JM. Hip-muscle activation during the lunge, single-leg squat, and step-up-and-over exercises. $J$ Sport Rehabil. 2009;18:91-103.

9. Distefano LJ, Blackburn JT, Marshall SW, Padua DA. Gluteal muscle activation during common therapeutic exercises. J Orthop Sports Phys Ther. 2009;39:532-540.

10. Ekstrom RA, Donatelli RA, Carp KC. Electromyographic analysis of core trunk, hip, and thigh muscles during 9 rehabilitation exercises. J Orthop Sports Phys Ther. 2007;37:754-762.

11. Escamilla RF, Babb E, DeWitt R, Jew P, Kelleher P, Burnham $\mathrm{T}$, et al. Electromyographic analysis of traditional and non-traditional abdominal exercises: implications for rehabilitation and training. Phys Ther. 2006;86:656-671.

12. O'Sullivan K, Smith SM, Sainsbury D. Electromyographic analysis of the three subdivisions of gluteus medius during weight-bearing exercises. Sports Med Arthrosc Rehabil Ther Technol. 2010;2:17-25. 
Copyright of Journal of Sport Rehabilitation is the property of Human Kinetics Publishers, Inc. and its content may not be copied or emailed to multiple sites or posted to a listserv without the copyright holder's express written permission. However, users may print, download, or email articles for individual use. 